\title{
How to estimate insulin resistance in PCOS patients - HOMA-IR or QUICKI?
}

Éva Csajbók*, Alexandra Gyói*, Sándor Magony*, Krisztián Sepp*, Zsuzsanna Valkusz* 1st Department of Internal Medicine, Endocrine Unit, University of Szeged, Hungary

Insulin resistance affects $50-70 \%$ of women with polycystic ovary syndrome (PCOS).

The aim of our study was to estimate the prevalence of insulin resistance by different methods in a single cohort of 63 PCOS patients diagnosed by the Rotterdam criteria

Methods: Anthropometric measurement, examination and fasting blood tests were made on the 3-5th days of their periods.

HOMA-IR (cut off $>2.5$ ) and QUICKI (cut off $<0.357$ ) was used to assess insulin resistance.

Results: Insulin resistant (IR) patients represented $48 \%$ and $65 \%$ of the cohort based on HOMA-IR (HIR) and QUICKI (QIR), respectively.

Compared to insulin sensitive (IS) patients, IR patients were older (25.5 \pm 5 vs. $30 \pm 6$ years).

The body mass index (BMI) was similar in the HIR $(35.5 \pm 7.33 \mathrm{~kg} / \mathrm{m} 2)$ and QIR $(33.15 \pm 7.81 \mathrm{~kg} / \mathrm{m} 2)$ and similar BMI was detected in IS patients according to HOMA (HIS, $25.7 \pm 4.74$ $\mathrm{kg} / \mathrm{m} 2)$ or QUICKI (QIS, $25.18 \pm 4.53 \mathrm{~kg} / \mathrm{m} 2)$.

Fasting glucose levels did not differ in between IR groups (HIR: $4.88 \pm 0.51$; QIR: $4.88 \pm 0.53 \mathrm{mmol} / \mathrm{l})$, but fasting insulin levels were higher in HIR (18.09 $\pm 8.05 \mathrm{mIU} / \mathrm{l})$ compared to QIR $(15.64 \pm 7.78 \mathrm{mlU} / \mathrm{l})$ patients.

Lipid profiles and HbA1c did not differ significantly between IR groups.

The LH:FSH ratio was higher in IR (HIR:2.89 \pm 1.44 , QIR:2.73 \pm 1.55 ) than in IS groups (HIS:2.11 \pm 1.12, QIS:2.38 \pm 1.21 )

Patients in IR groups had higher free androgen index (FAI) than IS patients (HIR: $8.64 \pm 6.4$, QIR: $8.48 \pm 6.21$, HIS:6.34 \pm 4.4 , QIS: $5.47 \pm 3.17$ ). OGTT was performed in 37 patients indicating neither diabetes nor IFG.

Conclusion: By using QUICKI we found more IR patient than with HOMA. Fasting insulin levels and BMI were lower in the QIR than in the HIR group. The FAI was similarly elevated in the IR groups, and was lowest in the QIS group.

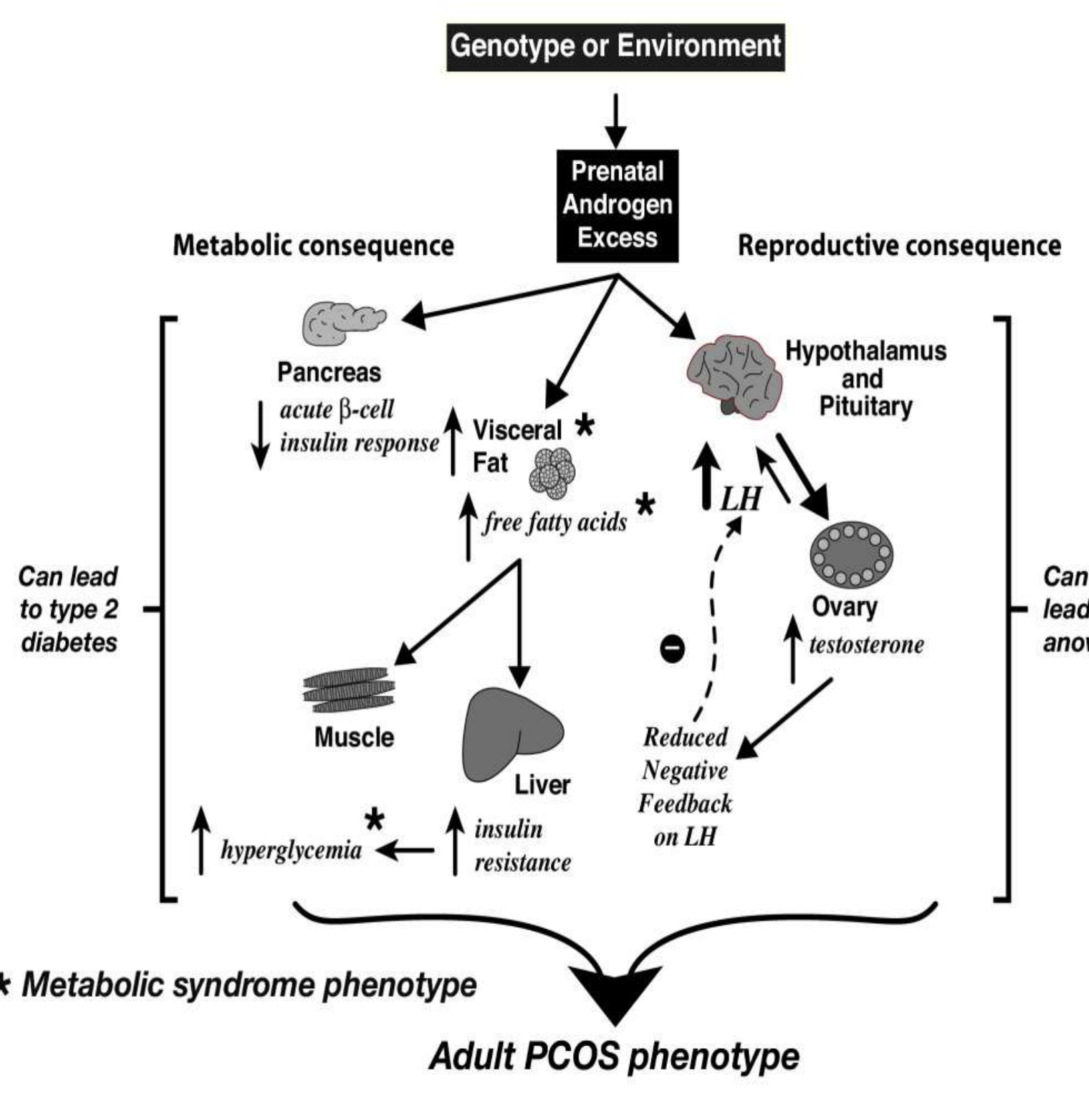

Our data

$\mathbf{N}=\mathbf{7 7}$ single cohort form 1 center

PCOS was diagnosed based on the Rotterdam criteria

Insulinresistance was calculated in 63 patients

Based on HOMA-IR and QUICKI we divided the patients into $\mathbf{4}$ groups:

can least

HIS: insulinsensitve based on HOMA-IR

QIR: insulinresistant based on QUICKI

QIS: insulinsensitive based on QUICKI

OGTT (oral glucose tolerance test, $75 \mathrm{~g}$ ) was made in 37 patients

\section{Conclusions}

By using QUICKI we found more IR patient than with HOMA

Fasting insulin levels and BMI were lower in the QIR than in the HIR group

The FAI was similarly elevated in the IR

groups, and was lowest in the QIS group

We suggest that QUICKI detects insulinresistance earlier than HOMA
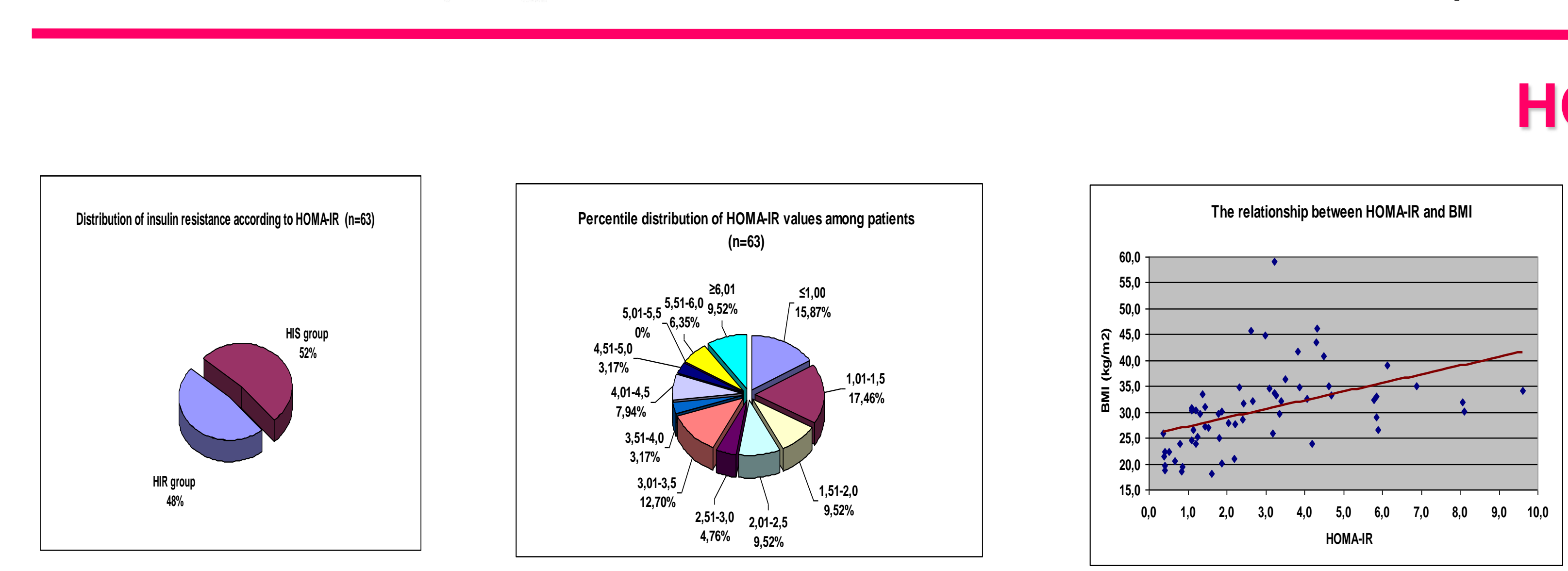

HOMA IR results
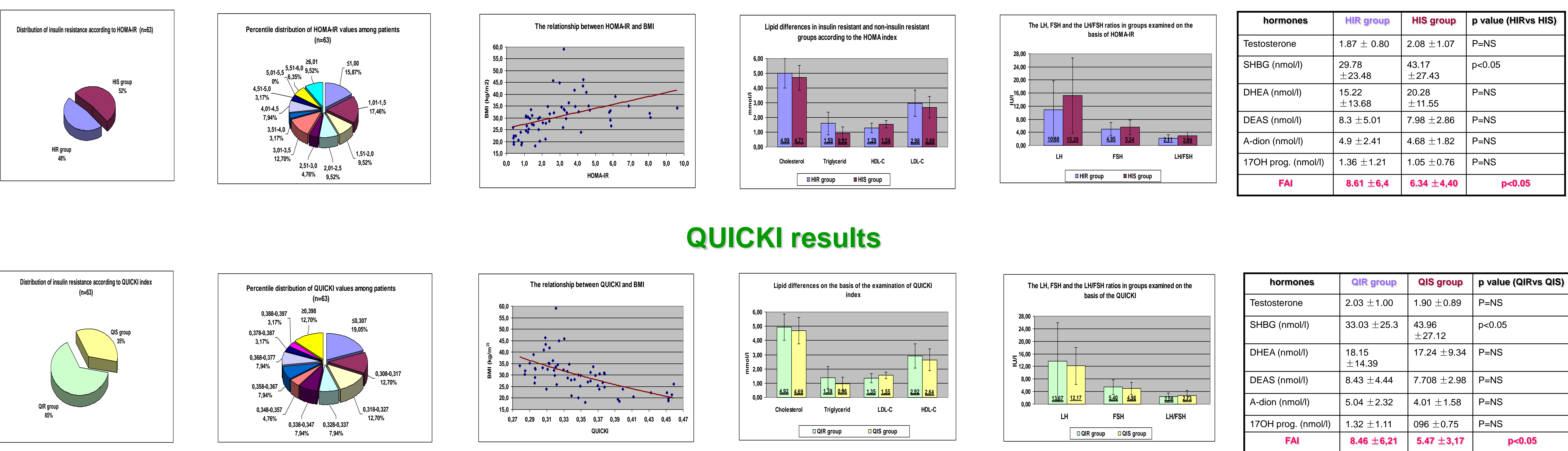

QUICKI results
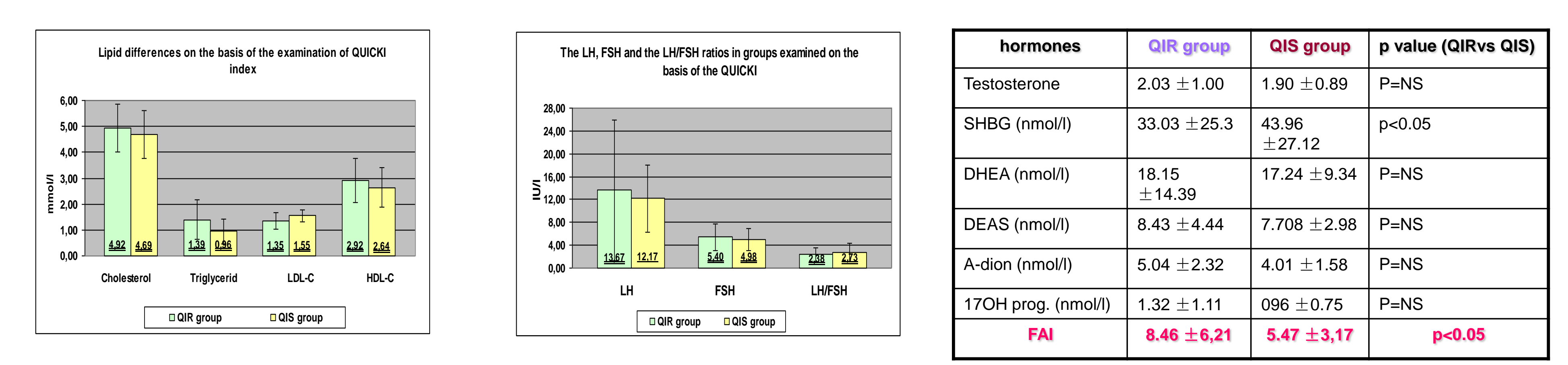

$\mathrm{LH} / \mathrm{FSH}$ ratio and FAl in insulinresistant patients
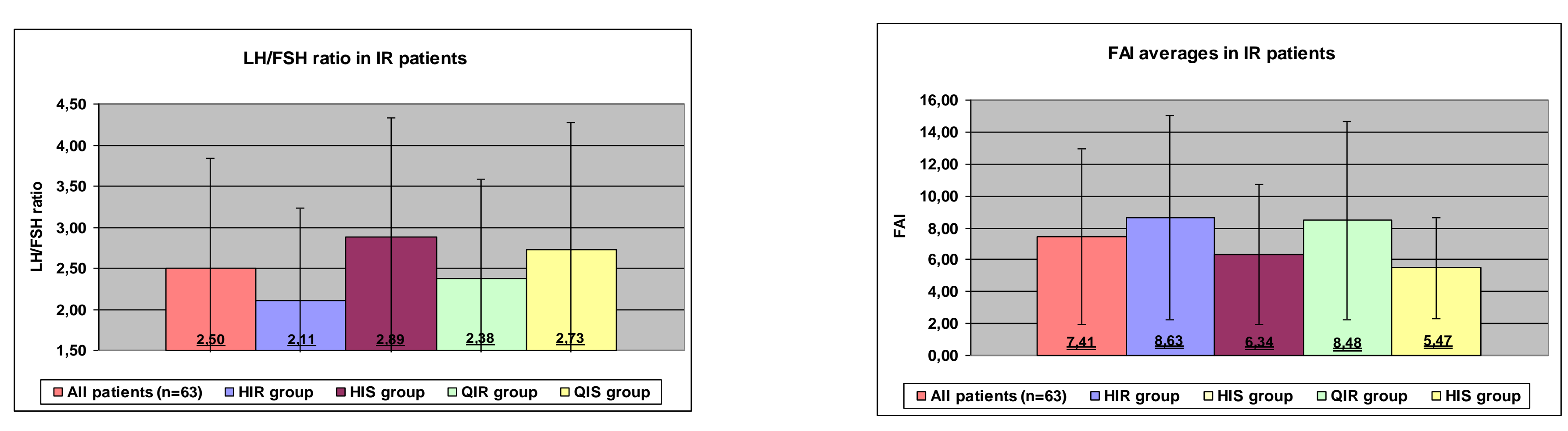

\section{OGTT results}
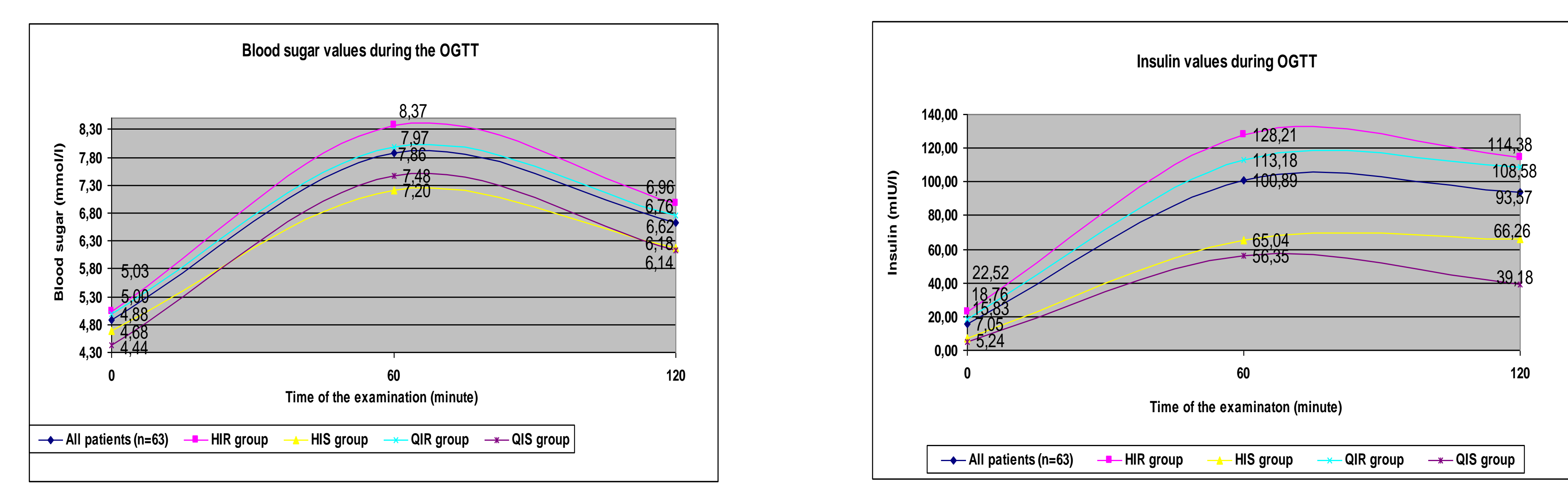